PROCEEDINGS OF THE

AMERICAN MATHEMATICAL SOCIETY

Volume 134, Number 9, September 2006, Pages 2677-2683

S 0002-9939(06)08302-X

Article electronically published on March 23, 2006

\title{
THE BANACH ALGEBRA GENERATED BY A CONTRACTION
}

\author{
H. S. MUSTAFAYEV
}

(Communicated by Joseph A. Ball)

\begin{abstract}
Let $T$ be a contraction on a Banach space and $A_{T}$ the Banach algebra generated by $T$. Let $\sigma_{u}(T)$ be the unitary spectrum (i.e., the intersection of $\sigma(T)$ with the unit circle) of $T$. We prove the following theorem of Katznelson-Tzafriri type: If $\sigma_{u}(T)$ is at most countable, then the Gelfand transform of $R \in A_{T}$ vanishes on $\sigma_{u}(T)$ if and only if $\lim _{n \rightarrow \infty}\left\|T^{n} R\right\|=0$.
\end{abstract}

Let $X$ be a complex Banach space $B(X)$, the algebra of all bounded linear operators on $X$, and let $I$ be the identity operator on $X . \sigma(T)$ will denote the spectrum of an operator $T \in B(X)$, and $R_{z}(T)=(z-T)^{-1}$ will denote the resolvent of $T$. If $A$ is a uniformly closed subalgebra of $B(X)$ with identity $I$, then $\sigma_{A}(T)$ will denote the spectrum of $T \in A$ with respect to $A$. If $T \in B(X)$, by $A_{T}$ we will denote the uniformly closed subalgebra of $B(X)$ generated by $T$ and $I$. $A_{T}$ is a commutative unital Banach algebra. As is well known, the maximal ideal space of $A_{T}$ can be identified with $\sigma_{A_{T}}(T) . \hat{R}$ will denote the Gelfand transform of any $R \in A_{T}$.

Let $T$ be a contraction (i.e., a linear operator of norm $\leq 1$ ) on a Banach space $X$. Then for every $x \in X$ the $\operatorname{limit}_{n \rightarrow \infty}\left\|T^{n} x\right\|$ exists and is equal to $\inf _{n \in \mathbb{N}}\left\|T^{n} x\right\|$. Note also that $\sigma(T) \subset \sigma_{A_{T}}(T) \subset \bar{D} ; D=\{z \in \mathbb{C}:|z|<1\}$. Let $\Gamma$ be the unit circle. $\sigma_{u}(T)=\sigma(T) \cap \Gamma$ is called the unitary spectrum of $T$. It is easy to see that if $\sigma_{u}(T)=\varnothing$, then $\lim _{n \rightarrow \infty}\left\|T^{n}\right\|=0$.

It follows from the Y. Katznelson and L. Tzafriri Theorem [4, Theorem 5] that if $\sigma_{u}(T)$ is at most countable and $f \equiv 0$ on $\sigma_{u}(T)$, then $\lim _{n \rightarrow \infty}\left\|T^{n} f(T)\right\|=0$, and that $f(z)$ is a function analytic in $D$, which has absolutely convergent Taylor series. In this note we obtain the following extension of this result.

Theorem 1. Let $T$ be a contraction on a Banach space such that the unitary spectrum $\sigma_{u}(T)$ of $T$ is at most countable. Then the Gelfand transform of $R \in A_{T}$ vanishes on $\sigma_{u}(T)$ if and only if $\lim _{n \rightarrow \infty}\left\|T^{n} R\right\|=0$.

For the proof we need some preliminary results.

The proof of the following lemma is similar to that of [7, Lemma 2.1].

Lemma 1. Let $T$ be a contraction on a Banach space $X$ such that $\sigma(T) \neq \bar{D}$ and $\inf _{n \in \mathbb{N}}\left\|T^{n} x\right\|>0$ for some $x \in X \backslash\{0\}$. Then there exist a Banach space $Y \neq\{0\}$, a bounded linear operator $J: X \rightarrow Y$ with dense range and a surjective isometry $S$

Received by the editors February 25, 2005 and, in revised form, April 5, 2005.

2000 Mathematics Subject Classification. Primary 47Axx.

Key words and phrases. Contraction, Banach algebra, spectrum, semisimplicity.

(C)2006 American Mathematical Society 
on $Y$ such that:

(i) $\|J x\|=\lim _{n \rightarrow \infty}\left\|T^{n} x\right\|$;

(ii) $S J=J T$;

(iii) $\sigma(S) \subset \sigma(T)$.

Proof. On $X$ we define the semi-norm $p$ by $p(x)=\lim _{n \rightarrow \infty}\left\|T^{n} x\right\|$. Put $E=\operatorname{ker} p$. Then $E$ is a closed invariant subspace of $T$ and $E \neq X$. Let $J: X \rightarrow X / E$ be the quotient mapping. Then the semi-norm $p$ induces a norm $\bar{p}$ on $Y_{0}=X / E$ by $\bar{p}(J x)=p(x)$, and we have $\|J x\|=\lim _{n \rightarrow \infty}\left\|T^{n} x\right\|$. Let $Y$ be the completion of $Y_{0}$ with respect to the norm $\bar{p}$. Define $S_{0}: Y_{0} \rightarrow Y_{0}$ by $S_{0} J=J T$. Since $\left\|S_{0} J x\right\|=\|J x\|, S_{0}$ extends to an isometry $S$ on $Y$. Then we have $S J=J T$, where $J: X \rightarrow Y$ has dense range.

Let $z \notin \sigma(T)$. From the obvious inequality $p\left(R_{z}(T) x\right) \leq\left\|R_{z}(T)\right\| p(x) \quad(x \in$ $X)$, it follows that $\sigma(S) \subset \sigma(T)$. If $S$ is a non-surjective isometry, then $\sigma(S)=\bar{D}$ [1. p. 27], and therefore $\sigma(T)=\bar{D}$. This contradicts $\sigma(T) \neq \bar{D}$. Hence, $S$ is a surjective isometry. The proof is complete.

If $T$ is a surjective isometry on a Banach space $X$, then $\sigma(T) \subset \Gamma$ and

$$
R_{z}(T)=\left\{\begin{array}{cl}
\sum_{n=0}^{\infty} z^{-n-1} T^{n}, & |z|>1, \\
-\sum_{n=1}^{\infty} z^{n-1} T^{-n}, & |z|<1 .
\end{array}\right.
$$

It follows that $\left\|R_{z}(T)\right\| \leq|| z|-1|^{-1}(|z| \neq 1)$. Now let $f \in L^{1}(\mathbb{Z})$ and

$$
\hat{f}(\xi)=\sum_{n \in \mathbb{Z}} f(n) \bar{\xi}^{n} \quad(\xi \in \Gamma),
$$

the Fourier transform of $f$. We can define $\hat{f}(T) \in B(X)$ by

$$
\hat{f}(T)=\sum_{n \in \mathbb{Z}} f(n) T^{-n} .
$$

Lemma 2. Let $T$ be a surjective isometry on a Banach space and let $f \in L^{1}(\mathbb{Z})$. If $\hat{f}(\xi)=0$ in a neighborhood of $\sigma(T)$, then $\hat{f}(T)=0$.

Proof. Let $U$ be an open set in $\Gamma$ that contains $\sigma(T)$. Assume that $\hat{f}$ vanishes on $U$. Then we have

$$
\begin{aligned}
\hat{f}(T)=\lim _{r \rightarrow 1^{-}} & \sum_{n \in \mathbb{Z}} r^{|n|} f(n) T^{-n}=\lim _{r \rightarrow 1^{-}} \int_{\Gamma} \hat{f}(\xi)\left(\sum_{n \in \mathbb{Z}} r^{|n|} \xi^{n} T^{-n}\right) d \xi \\
& =\lim _{r \rightarrow 1^{-}} \int_{\Gamma-U} \hat{f}(\xi)\left(T R_{r^{-1} \xi}(T)-T R_{\xi}(T)\right) d \xi \\
& +\lim _{r \rightarrow 1^{-}} \int_{\Gamma-U} \hat{f}(\xi)\left(T R_{\xi}(T)-T R_{r \xi}(T)\right) d \xi=0 .
\end{aligned}
$$

We will also need the following notation.

Recall that $\varphi=\{\varphi(n)\}_{n \in \mathbb{Z}} \in L^{\infty}(\mathbb{Z})$ is almost periodic on $\mathbb{Z}$ if $\left\{\varphi_{m}: m \in \mathbb{Z}\right\}$ is relatively compact in the norm topology of $L^{\infty}(\mathbb{Z})$, where $\varphi_{m}(n)=\varphi(n+m)$. We denote by $A P(\mathbb{Z})$ the set of all almost periodic functions on $\mathbb{Z} . A P(\mathbb{Z})$ is a closed 
subalgebra of $L^{\infty}(\mathbb{Z})$. As is well known, there exists a unique $\Phi \in A P(\mathbb{Z})^{*}$ (which is called invariant mean on $A P(\mathbb{Z})$ ) such that:

(i) $\Phi(\mathbf{1})=1$, where $\mathbf{1}(n) \equiv 1$;

(ii) $\Phi(\varphi) \geq 0$ for all $\varphi \geq 0$;

(iii) $\Phi\left(\varphi_{m}\right)=\Phi(\varphi)$ for all $\varphi \in L^{\infty}(\mathbb{Z})$ and $m \in \mathbb{Z}$.

The hull of any ideal $I \subset L^{1}(\mathbb{Z})$ is $h(I)=\{\xi \in \Gamma: \hat{f}(\xi)=0, f \in I\}$. For a closed subset $K \subset \Gamma$, let $I_{K}=\left\{f \in L^{1}(\mathbb{Z}): \hat{f}(K)=\{0\}\right\}$ and $J_{K}^{0}=\left\{f \in L^{1}(\mathbb{Z})\right.$ : supp $\hat{f} \cap K=\varnothing\}$. $K$ is a set of synthesis if $I_{K}=\overline{J_{K}^{0}}$. For example, closed countable sets are sets of synthesis. As is well known (Malliavin's theorem), there exists a non-synthesis set (see [5, chap. 8]).

For $\varphi \in L^{\infty}(\mathbb{Z})$ and $f \in L^{1}(\mathbb{Z}), \varphi * f$ will denote the convolution of $\varphi$ and $f$. Recall that the $w^{*}$-spectrum $\sigma_{*}(\varphi)$ of $\varphi \in L^{\infty}(\mathbb{Z})$ is defined as the hull of the closed ideal $I_{\varphi}=\left\{f \in L^{1}(\mathbb{Z}): \varphi * f=0\right\}$. The well-known theorem of Loomis [6 states that if the $w^{*}$-spectrum of $\varphi \in L^{\infty}(\mathbb{Z})$ is at most countable, then $\varphi \in A P(\mathbb{Z})$.

Lemma 3. Let $S$ be a surjective isometry on a Banach space $Y$ such that $\sigma(S)$ is at most countable. Then for every $\phi \in Y^{*}$, there exists a Hilbert space $H_{\phi}$, a bounded linear operator $J_{\phi}: Y \rightarrow H_{\phi}$ with dense range and a unitary operator $U_{\phi}$ on $H_{\phi}$ such that:

(i) $U_{\phi} J_{\phi}=J_{\phi} S$;

(ii) $\sigma\left(U_{\phi}^{*}\right) \subset \sigma(S)$.

Proof. (i) Let $\phi \in Y^{*}$. For given $y \in Y$, define the function $\bar{y}_{\phi}$ on $\mathbb{Z}$ by $\bar{y}_{\phi}(n)=$ $\phi\left(S^{n} y\right)$. Since $\left\|\bar{y}_{\phi}\right\|_{\infty} \leq\|\phi\|\|y\|, \bar{y}_{\phi}$ is a bounded function. We claim that $\sigma_{*}\left(\bar{y}_{\phi}\right) \subset$ $\sigma(S)$. Assume that there exists a $\xi_{0} \in \sigma_{*}\left(\bar{y}_{\phi}\right)$, but $\xi_{0} \notin \sigma(S)$. Then there exists an $f \in L^{1}(\mathbb{Z})$ such that $\hat{f}\left(\xi_{0}\right) \neq 0$ and $\hat{f}(\xi)=0$ on some neighborhood of $\sigma(S)$.

By Lemma $2, \hat{f}(S)=0$ and consequently,

$$
0=\phi\left(S^{n} \hat{f}(S) y\right)=\left(\bar{y}_{\phi} * f\right)(n), \quad \text { for all } n \in \mathbb{Z} .
$$

Since $\xi_{0} \in \sigma_{*}\left(\bar{y}_{\phi}\right)$ it follows that $\hat{f}\left(\xi_{0}\right)=0$. This contradiction proves the claim. Hence, $\sigma_{*}\left(\bar{y}_{\phi}\right)$ is at most countable. By the Loomis Theorem $\left[\underline{6}, \bar{y}_{\phi} \in A P(\mathbb{Z})\right.$.

Let $H_{\phi}^{0}$ denote the linear set $\left\{\bar{y}_{\phi}: y \in Y\right\}$ with the inner product defined by

$$
\left\langle\bar{y}_{\phi}, \bar{z}_{\phi}\right\rangle=\Phi\left(\left\{\bar{y}_{\phi}(n) \overline{\bar{z}_{\phi}(n)}\right\}_{n \in \mathbb{Z}}\right), \quad z \in Y
$$

where $\Phi$ is the invariant mean on $A P(\mathbb{Z})$. Let $H_{\phi}$ be the completion of $H_{\phi}^{0}$ with respect to the norm

$$
\left\|\bar{y}_{\phi}\right\|_{2}^{2}=\Phi\left(\left\{\left|\bar{y}_{\phi}(n)\right|^{2}\right\}_{n \in \mathbb{Z}}\right) .
$$

Then $H_{\phi}$ is a Hilbert space. Note also that $\left\|\bar{y}_{\phi}\right\|_{2} \leq\left\|\bar{y}_{\phi}\right\|_{\infty} \leq\|\phi\|\|y\|$. It follows that the map $J_{\phi}: Y \rightarrow H_{\phi}$, defined by $J_{\phi} y=\bar{y}_{\phi}$, is a bounded linear operator with dense range. Now define the map $U_{\phi}: H_{\phi} \rightarrow H_{\phi}$, by $U_{\phi} \bar{y}_{\phi}=(\overline{S y})_{\phi}$. It is easy to see that $U_{\phi}$ is a unitary operator and $U_{\phi} J_{\phi}=J_{\phi} S$. We have proved (i).

Next we prove (ii). We have

$$
S^{*} J_{\phi}^{*}=J_{\phi}^{*} U_{\phi}^{*}
$$


Assume that there exists $\xi \in \sigma\left(U_{\phi}^{*}\right)$, but $\xi \notin \sigma(S)=\sigma\left(S^{*}\right)$. Put $\delta=\left\|\left(S^{*}-\xi\right)^{-1}\right\|^{-1}$. Choose $\varepsilon>0$ such that $\varepsilon<\delta$. Let $\Gamma_{\varepsilon}=\{z \in \mathbb{C}:|z-\xi|<\varepsilon\} \cap \Gamma$ and let $E(\cdot)$ be the spectral measure for $U_{\phi}^{*}$. Since $\sigma\left(U_{\phi}^{*}\right) \cap \Gamma_{\varepsilon} \neq \varnothing$, we have $E\left(\Delta_{\varepsilon}\right) \neq 0$. Let $h \in E\left(\Delta_{\varepsilon}\right) H_{\phi}$ be such that $\|h\|=1$. From the identity

$$
\left(U_{\phi}^{*}-\xi\right)^{n} h=\int_{\Gamma_{\varepsilon}}(t-\xi)^{n} d E(t) h, \quad n \in \mathbb{N},
$$

we have

$$
\left\|\left(U_{\phi}^{*}-\xi\right)^{n} h\right\| \leq \varepsilon^{n} .
$$

On the other hand from (11) we can write

$$
\left(S^{*}-\xi\right)^{n} J_{\phi}^{*} h=J_{\phi}^{*}\left(U_{\phi}^{*}-\xi\right)^{n} h .
$$

It follows that

$$
\left\|\left(S^{*}-\xi\right)^{n} J_{\phi}^{*} h\right\| \leq \varepsilon^{n}\left\|J_{\phi}^{*}\right\|
$$

Consequently, we have

$$
\left\|J_{\phi}^{*} h\right\| \leq\left\|\left(S^{*}-\xi\right)^{-n}\right\|\left\|\left(S^{*}-\xi\right)^{n} J_{\phi}^{*} h\right\| \leq\left(\frac{\varepsilon}{\delta}\right)^{n}\left\|J_{\phi}^{*}\right\| \rightarrow 0, \text { as } n \rightarrow \infty .
$$

Hence, $J_{\phi}^{*} h=0$. Since $J_{\phi}^{*}$ has zero kernel, we obtain $h=0$. This is a contradiction. The proof is complete.

Proof of Theorem 1. Let $R \in A_{T}$. Assume that $\lim _{n \rightarrow \infty}\left\|T^{n} R\right\|=0$. Then for any $\xi \in \sigma_{u}(T),\left|\hat{T}^{n}(\xi) \hat{R}(\xi)\right| \rightarrow 0$, as $n \rightarrow \infty$. Since $|\hat{T}(\xi)| \equiv 1$, it follows that $\hat{R}(\xi)=0$. Now assume that $\sigma_{u}(T)$ is at most countable and $\hat{R}(\xi) \equiv 0$ on $\sigma_{u}(T)$. It is enough to prove that $\lim _{n \rightarrow \infty}\left\|T^{n} R x\right\|=0$ for all $x \in X$. Indeed, suppose that this is proved. For $C \in B(X)$, let $L_{C}$ be the left multiplication operator on $B(X)$ defined by $L_{C} F=C F$. Then $L_{T}$ is a contraction. Moreover, the maximal ideal spaces of $A_{L_{T}}$ and $A_{T}$ are the same, and $\sigma\left(L_{T}\right)=\sigma(T)$. Note also that $L_{R} \in A_{L_{T}}$ and the Gelfand transform of $L_{R}$ vanishes on $\sigma_{u}\left(L_{T}\right)$. Therefore,

$$
\lim _{n \rightarrow \infty}\left\|L_{T}^{n} L_{R} F\right\|=0,
$$

for all $F \in B(X)$. Taking $F=I$, we get the desired conclusion.

If $\lim _{n \rightarrow \infty}\left\|T^{n} x\right\|=0$ for all $x \in X$, then there is nothing to prove. Hence, we may assume that $\lim _{n \rightarrow \infty}\left\|T^{n} x\right\|>0$ for some $x \neq 0$. On the other hand, since $\sigma_{u}(T)$ is at most countable, $\sigma(T) \neq \bar{D}$. In view of Lemma 1 there exists a Banach space $Y \neq\{0\}$, a bounded linear operator $J: X \rightarrow Y$ with dense range and a surjective isometry $S$ on $Y$ such that:

(i) $\|J x\|=\lim _{n \rightarrow \infty}\left\|T^{n} x\right\|$;

(ii) $S J=J T$;

(iii) $\sigma(S) \subset \sigma(T)$.

It follows from (iii) that $\sigma(S) \subset \sigma_{u}(T)$ and therefore, $\sigma(S)$ is at most countable. Now let $\phi \in Y^{*}$ be given. By Lemma 3, there exists a Hilbert space $H_{\phi}$, a bounded linear operator $J_{\phi}: Y \rightarrow H_{\phi}$ with dense range and a unitary operator $U_{\phi}$ on $H_{\phi}$ such that

$$
U_{\phi} J_{\phi}=J_{\phi} S
$$


and $\sigma\left(U_{\phi}^{*}\right) \subset \sigma(S) \subset \sigma_{u}(T)$. Moreover, from (ii) and (2) we obtain

$$
U_{\phi} J_{\phi} J=J_{\phi} J T
$$

Further, since $R \in A_{T}$, there exists a sequence of polynomials $\left\{P_{n}(z)\right\}_{n \in \mathbb{N}}$ such that $\left\|P_{n}(T)-R\right\| \rightarrow 0$. Also since the Gelfand transform of $R$ vanishes on $\sigma_{u}(T)$, the sequence $\left\{P_{n}(z)\right\}_{n \in \mathbb{N}}$ uniformly converges to zero on $\sigma_{u}(T)$. Hence, the sequence $\left\{P_{n}(z)\right\}_{n \in \mathbb{N}}$ uniformly converges to zero on $\sigma\left(U_{\phi}^{*}\right)$. It follows that $\left\|P_{n}\left(U_{\phi}^{*}\right)\right\| \rightarrow 0$. On the other hand, from the identity (3) we can write

$$
J^{*} J_{\phi}^{*} P_{n}\left(U_{\phi}^{*}\right)=P_{n}\left(T^{*}\right) J^{*} J_{\phi}^{*}
$$

This clearly implies that $R^{*} J^{*} J_{\phi}^{*}=0$ and so $J_{\phi} J R=0$. Hence, $\phi(J R x)=0$ for all $\phi \in Y^{*}$ and $x \in X$. Thus, we obtain that

$$
0=\|J R x\|=\lim _{n \rightarrow \infty}\left\|T^{n} R x\right\|,
$$

for all $x \in X$. This completes the proof.

We do not know whether Theorem 1 true if $\sigma_{u}(T)$ is a synthesis set.

Remark 1. Theorem 1 remains valid if $\sup _{n \in \mathbb{N}}\left\|T^{n}\right\|<\infty$. Indeed, in this case $\||x|\|=\sup _{n \in \mathbb{N}}\left\|T^{n} x\right\|$ is an equivalent norm on $X$ with respect to which $T$ becomes a contraction.

In contrast with the unitary operator on a Hilbert space, there exists a surjective isometry on a Banach space that generates a non-semisimple algebra (see [3]). But surjective isometry on a Banach space with countable spectrum generated a semisimple algebra [3]. The following example shows that even on a Hilbert space there exists a contraction with countable unitary spectrum that generates a non-semisimple algebra: Let $V$ be the Volterra operator on $L^{2}[0,1]$ defined by $(V f)(t)=\int_{0}^{t} f(s) d s$ and let $T=(I+V)^{-1}$. Then $\left\|T^{n}\right\|=1$ for all $n \in \mathbb{N}$ and $\sigma(T)=\{1\}$. But $T \neq I$.

Recall that a contraction $T$ on a Banach space $X$ is said to be a $C_{1}$-contraction if $\inf _{n \in \mathbb{N}}\left\|T^{n} x\right\|>0$ for all $x \in X \backslash\{0\}$ [1, p. 250].

Corollary 1. Let $T$ be a $C_{1}$-contraction on a Banach space such that the unitary spectrum $\sigma_{u}(T)$ of $T$ is at most countable. If the Gelfand transform of $R \in A_{T}$ vanishes on $\sigma_{u}(T)$, then $R=0$. In particular, $A_{T}$ is semisimple.

For contractions on a Hilbert space, the Katznelson-Tzafriri theorem can be improved as follows [2]: If $T$ is a contraction on a Hilbert space and $f \in A(D)$ vanishes on $\sigma_{u}(T)$, then

$$
\lim _{n \rightarrow \infty}\left\|T^{n} f(T)\right\|=0
$$

$A(D)$ is the disc algebra. Now let $R \in A_{T}$ be such that $\hat{R}(\xi) \equiv 0$ on $\sigma_{u}(T)$. Is it then true that $\lim _{n \rightarrow \infty}\left\|T^{n} R\right\|=0$ ? We do not know the answer to this question.

However, we prove the following.

Theorem 2. Let $H$ be a Hilbert space and let $T$ be a contraction on $H$. If the Gelfand transform of $R \in A_{T}$ vanishes on $\sigma_{u}(T)$, then

$$
\lim _{n \rightarrow \infty}\left\|T^{n} R x\right\|=0
$$

for all $x \in H$. 
Proof. Let $R \in A_{T}$ be such that $\hat{R}(\xi) \equiv 0$ on $\sigma_{u}(T)$. We define a new inner product on $H$ by the formula

$$
[x, y]=\lim _{n \rightarrow \infty}\left\langle T^{n} x, T^{n} y\right\rangle
$$

(it is easy to see that the limit on the right-hand side exists). This induced a semi-norm on $H$ defined by

$$
p(x)=\left(\lim _{n \rightarrow \infty}\left\|T^{n} x\right\|^{2}\right)^{1 / 2} .
$$

Let $E=\operatorname{ker} p$. It is clear that $E$ is a closed invariant subspace of $T$. If $E=H$, then there is nothing to prove. Hence, we may assume that $E \neq H$. Let $J: H \rightarrow H / E$ be the quotient mapping. Then the semi-norm $p$ induces a norm $\bar{p}$ on $K_{0}=H / E$ by $\bar{p}(J x)=p(x)$, and we have

$$
\|J x\|=\left(\lim _{n \rightarrow \infty}\left\|T^{n} x\right\|^{2}\right)^{1 / 2} .
$$

Let $K$ be the completion of $K_{0}$ with respect to the norm $\bar{p}$. Define $U_{0}: K_{0} \rightarrow K_{0}$ by $U_{0} J=J T$. Since $\left\|U_{0} J x\right\| \leq\|T\|\|J x\|, U_{0}$ extends to a bounded operator $U$ on $K$. Then we have $U J=J T$, where $J: H \rightarrow K$ has dense range. Also since

$$
[U p(x), U p(y)]=[p(x), p(y)], \quad x, y \in H,
$$

$U$ is an isometry on $K$. As in the proof of Lemma 1 we can see that $\sigma(U) \subset \sigma(T)$.

Now assume that $U$ is a non-surjective isometry. Then $\sigma(U)=\bar{D}$ and consequently, $\sigma(T)=\bar{D}$. Hence, $\sigma_{u}(T)=\Gamma$. Since $R \in A_{T}$, there exists a sequence $\left\{P_{n}(z)\right\}_{n \in \mathbb{N}}$ of polynomials such that $\left\|P_{n}(T)-R\right\| \rightarrow 0$. It follows that $P_{n}(z) \rightarrow 0$ uniformly on $\Gamma$. By the von Neumann inequality,

$$
\left\|P_{n}(T)\right\| \leq \sup _{\xi \in \Gamma}\left|P_{n}(\xi)\right| \rightarrow 0,
$$

and so $R=0$. Hence, we may assume that $U$ is a unitary operator. As above, there exists a sequence $\left\{P_{n}(z)\right\}_{n \in \mathbb{N}}$ of polynomials such that $\left\|P_{n}(T)-R\right\| \rightarrow 0$. It follows that $P_{n}(z) \rightarrow 0$ uniformly on $\sigma_{u}(T)$. Since $\sigma(U) \subset \sigma_{u}(T)$, we have $\left\|P_{n}(U)\right\| \rightarrow 0$. Now from the identity $P_{n}(U) J=J P_{n}(T)$ we obtain that $J R=0$. Hence we have that $\lim _{n \rightarrow \infty}\left\|T^{n} R x\right\|=0$ for all $x \in H$. The proof is complete.

A similar result holds for the power-bounded operators.

Theorem 3. Let $T$ be a power-bounded operator on a Hilbert space $H$. If the Gelfand transform of $R \in A_{T}$ vanishes on $\sigma_{u}(T)$, then for all $x \in H$,

$$
\text { l.i.m. }\left\|T^{n} R x\right\|=0,
$$

where l.i.m. is a Banach limit on $\mathbb{N}$.

Corollary 2. Let $T$ be a contraction on a Hilbert space. If $R \in A_{T}$ is a compact operator and $\hat{R}(\xi) \equiv 0$ on $\sigma_{u}(T)$, then

$$
\lim _{n \rightarrow \infty}\left\|T^{n} R\right\|=0 .
$$




\section{REFERENCES}

[1] A. Beauzamy, Introduction to Operator Theory and Invariant Subspaces, North-Holland, Amsterdam, 1988. MR0967989 (90d:47001)

[2] J. Esterle, E. Strouse and F. Zouakia, Theorems of Katznelson-Tzafriri type for contractions, J. Funct. Anal., 94(1990), 273-287. MR1081645 (92c:47016)

[3] G. M. Feldman, The semisimplicity of an algebra generated by isometric operators, Funksional Anal. Prilozhen., 8(1974), 93-94 (Russian). MR0361800(50:14245)

[4] Y. Katznelson and L. Tzafriri, On power bounded operators, J. Funct. Anal., 68(1986), 313-328. MR.0859138 (88e:47006)

[5] R. Larsen, Banach Algebras, Marcel Dekker, New York, 1973. MR0487369 (58:7010)

[6] L. H. Loomis, The spectral characterization of a class of almost periodic functions, Ann. Math., 72(1960), 362-368. MR0120502 (22:11255)

[7] Vu Quoc Phong, Theorems of Katznelson-Tzafriri type for temigroups of operators, J. Funct. Anal., 103(1992), 74-84. MR.1144683 (93e:47050)

Department of Mathematics, Faculty of Arts and Sciences, Yüzüncü Yil University, 65080, VAN, TURKEY

E-mail address: hsmustafayev@yahoo.com 